\title{
Analysis of the Spatial-Temporal Distribution of Dengue Case Notifications in the Municipality of Vitória de Santo Antão-PE
}

\author{
Andrezza Fabianni Pedrosa dos Santos Lima1, Emilly Tainá Batista da Silva ${ }^{2}$, Emivaldo Batista da \\ Silva $^{3 *}$, Joana Bulhões Alvares da Silva Lima ${ }^{4}$, Ranyere Maria de Melo Silva ${ }^{5}$, Guilherme Expedito \\ Rosendo da Silva ${ }^{6}$, Josivan de Souza Vilanova Neto ${ }^{7}$, Suelane Michelly Ferreira Alves ${ }^{8}$, Jessikele \\ Gonçalves Bezerra9 ${ }^{9}$, Valéria Bezerra da Silva ${ }^{10}$, Ana Greice Borba Leite ${ }^{11}$, Emanuella Souza Bacelar ${ }^{12}$, \\ Simara Lopes Cruz Damazio ${ }^{13}$, Islane Cristina Martins ${ }^{14}$, Antônio Flaudiano Bem Leite ${ }^{15}$, Emanuella \\ Barros de Souza Oliveira Alvares ${ }^{16}$
}

1,5,6,7,8,9,10 Department of Nutrition, University Center of Vitória de Santo Antão - UNIVISA, Vitoria de Santo Antão, Brazil

2 Department of Biomedicine, Post-Graduation in Laboratory Clinical Microbiology (ASCES- UNITA) Caruaru - PE

3,4,12 Department of Pharmaceutical Sciences, University Center of Vitória de Santo Antão - (UNIVISA) Vitória de Santo Antão, Brazil.

11 Department of Veterinary Medicine Rural University of Pernambuco, Recife - PE

13 Department of Bachelor of Nursing Federal University of Pernambuco, Vitória de Santo Antão, PE.

14.15 Department of Collective Health Federal University of Pernambuco, Vitória de Santo Antão, PE, Brazil.

16 Department of Biology, Center for Biological Sciences, Professor of the Degree course in Biology, University Center of Vitória de Santo Antão (UNIVISA), Vitória de Santo Antão, Brazil

E-mail adresses: andrezzafpslima@hotmail.com1 (Andrezza Fabianni Pedrosa dos Santos Lima), emilly.taina@hotmail.com2 (Emilly Tainá Batista da Silva), emivaldobatista4@gmail.com3 (Emivaldo Batista da Silva), joanafarmacia2018@gmail.com4 (Joana Bulhões Alvares da Silva Lima), ranyymelo@gmail.com5 (Ranyere Maria de Melo Silva),guilhermenutri1@gmail.com6 (Guilherme expedito Rosendo da Silva), neto.com.br2015@hotmail.com 7 (Josivan de Sousa Vilanova Neto), llanealves82@gmail.com8 (Suelane Michelly Ferreira Alves), jessikeleg@gmail.com9 (Jessikele Gonçalves Bezerra), valeriabezerra.s@hotmail.com10 (Valéria Bezerra da Silva), ag_mv530@hotmail.com11 (Ana Greice Borba Leite), mannubacelar@gmail.com12 (Emanuella Souza Bacelar), simara.cruz@ufpe.br13 (Simaria Lopes Cruz Damazio), islanemartins@gmail.com14 (Islane Cristina Martins), afbl@outlook.com.br15 (Antônio Flaudiano Bem Leite), emanuella.barros@hotmail.com16 (Emanuella Barros de Souza Oliveira Alvares)

${ }^{*}$ Corresponding author

\section{To cite this article:}

Lima, A.F.P.S.; Silva, E.T.B.; Silva, E.B.; Lima, J.B.A.S.; Silva, R.M.M.; Silva, G.E.R.; Neto, J.S.V.; Alves, S.M.F.; Bezerra, J.G.; Silva, V.B.; Leite, A.G.B.; Bacelar, E.S.; Damazio, S.L.C.; Martins, I.C.; Leite, A.F.B.; Álvares, E.B.S.O. Analysis of the Spatial-Temporal Distribution of Dengue Case Notifications in the Municipality of Vitória de Santo Antão-PE. International Journal of Sciences. Vol. 3, No. 1, 2022, pp.4450. ISSN 2763-5392.

Received: 11 24, 2021; Accepted: 12 26, 2021; Published: 01 15, 2022

\begin{abstract}
The year 2015 was considered the most epidemic regarding dengue disease in Brazil, a disease that contributes with considerable socioeconomic influence in the communities of areas indicated as endemic, affecting all social levels. This study aimed to analyze the spatial-temporal distribution of dengue case notifications in the municipality of Vitória de Santo Antão-PE. A descriptive and epidemiological study of the spatial distribution of dengue cases was conducted in the municipality of Vitória de Santo Antão in 2015. The description of the control case respected the categorization offered by the Health Surveillance Guide published in 2014 effective until the beginning of 2016 and sinan's online dictionary. As results,2,203 dengue cases were reported in the municipality of Vitória de Santo Antão, among them dengue fever was confirmed in 128, confirmation for the clinicalepidemiological criterion in 1,027; these being: economically active population (n: 857 ); economically non-active population (n: 515); economically active male population (n: 432); economically non-active male population (n: 242); economically active female population (n: 425); economically non-active female population (n: 273); black population (n: 1,178); non-black population ( $\mathrm{n}=194)$; Rank level 1(n: 1,112) and Rank level 2 (n: 257). It is concluded that the analysis of the spatial-temporal
\end{abstract}


2 Lima, A.F.P.S.; Silva, E.T.B.; Silva, E.B.; Lima, J.B.A.S.; Silva, R.M.M.; Silva, G.E.R.; Neto, J.S.V.; Alves, S.M.F.; Bezerra, J.G.; Silva, V.B.; Leite, A.G.B.; Bacelar, E.S.; Damazio, S.L.C.; Martins, I.C.; Leite, A.F.B.; Alvares, E.B.S.O. Analysis of the Spatial-Temporal Distribution of Dengue Case Notifications in the Municipality of Vitória de Santo Antão-PE...

distribution of case notifications can direct public policies to more direct actions to control and combat the disease vector. The space-time approach allows the integration of demographic, economic and environmental data, promoting the interrelationship of information and various databases.

Keywords: Geoprocessing. Dengue fever. Epidemiology. Health Information Systems.

\section{Introduction}

Geoprocessing techniques and geostatistics have made it possible for ecological studies to make more evident the participation of the conjunction of factors, including contextual factors, in the determination of the disease by incorporating effects of the specific characteristics of each social space. Although with limitations, this approach contributes so that epidemiology can dialectically integrate the social with the natural, as supported by the thinkers of social epidemiology1.

According to Camargo et al. 2 The proper application of geoprocessing tools, enable the mapping of the areas most vulnerable to sanitary diseases, thus favoring the construction and management of public planning from the correct allocation of funds or funds to be used in public needs related to sanitation and infrastructure. It is noteworthy that the applicability of geoprocessing helps in the analysis and delineation of dengue control and combat, offering as a result accurate geospatial information 1 .

Spatial analysis has been applied for many years in exact sciences for the measurement of soil contamination dispersion, extension of mineral deposits and the variation of geoenvironmental characteristics of regionalized data3.

According to the World Health Organization (WHO), 2015 was the most epidemic in dengue disease in Brazil, surpassing the epidemiological records consolidated in previous years, 2010 and 2013, which was the period in which notifications presented a categorization pattern. Among the reemerging diseases, it is the one that constitutes the most serious public health problem in the country. Described as acute febrile disease, having a benign or severe course, depending on the form presented: dengue without alarm signs, dengue with alarm signs and severe dengue4,5.

However, the development of spatial analysis symbolized a valuable tool for understanding how a given context influences the quality or totality of the health of a community and population groups 1 .

Considering the various studies that demonstrate and affirm the relationship of environmental determinants and climatic factors under the dynamics of endemic smaemias, and knowing the pertinence and magnitude of dengue in Brazil and worldwide, the development of a systematic review of the literature becomes relevant. The present study aimed to perform an analysis of the spatial-temporal distribution of dengue notifications in the municipality of Vitória de Santo Antão - PE, inorder to understand how the temporal space of dengue cases is given.

\section{Methodology}

\section{Type of study}

A descriptive, epidemiological study of the populationbased spatial type (case-control) of the spatial distribution of dengue cases occurred in the municipality of Vitória de Santo Antão in 2015 was carried out.

\section{Study population}

The study population consisted of the reported cases of dengue in 2015, in the municipality of Vitoria de Santo Antão and consolidated in the Notifiable Diseases Information System.

\section{Inclusion and exclusion criteria}

The description of the control case respected the categorization offered by the Health Surveillance Guide published in 2014 effective until the beginning of 2016 and SINAN's online dictionary.

Inclusion criteria - The inclusion criteria of the research were defined by all reported cases of dengue in 2015, in the municipality of Vitória de Santo Antão and consolidated in the Notifiable Diseases Information System - SINAN. Being named as: Confirmed cases of dengue by epidemiological clinical investigation or confirmed cases of dengue by laboratory investigation.

Exclusion criteria - The exclusion criteria of the research were defined by all cases notificados of dengue in 2015, in the municipality of Vitória de Santo Antão and consolidated in the Notifiable Diseases Information System - SINAN. Being named as: Discarded cases of dengue and inconclusive cases.

\section{The area of study}

The municipality of Vitória de Santo Antão has a territorial area of approximately $335,942 \mathrm{~km}^{2}$, being $5.717 \mathrm{~km}^{2}$ in urban perimeter, with an estimated population of 136,706 inhabitants and a population density of 348.80 inhabitants $/ \mathrm{km}^{2}$, according to the population census of 2010 (IBGE, 2010).

\section{Statistical plan}

The data used in this study were obtained from the Municipal Health Department of Vitória de Santo Antão, referring to the reported cases of dengue in 2015. For the construction of the identification of dengue cases, the complete database of the Notifiable Diseases Information System (SINAN) was used.

To perform spatial statistics analyses, the Terra View program version 4.2.2 was applied.

Of the variables used in the reported cases of dengue were: dengue with laboratory confirmation; dengue with confirmation for the clinical - epidemiological criterion; with economically active population; economically non-active population; dengue in the economically active male 
population; dengue in the economically non-active male population; dengue in the economically active female population; dengue in the economically non-active female population; dengue in the black population; dengue in the nonblack population; dengue with Classification level 1 (Less and equal to 5 signs and symptoms, except signs and symptoms defined by the Ministry of Health as severe); dengue with Level 2 classification Greater than 5 symptoms or with severity bells and deaths). The reported cases of dengue were geocoded based on the address contained in the individual's notification form.

Each notified case of dengue was located geographically with the help of Google's google earth engine platform, free access, which allowed to manipulate and combine information obtained by remote sensing, on a planetary scale.

However, several cases were not found due to failures in filling out the forms referring to the patients' addresses, because some information was incomplete, such as the full address of residence

\section{Spatial analysis of dengue reported cases}

Spatial analysis emphasizes measuring properties and relationships, taking into account the geographic location of the phenomenon under study explicitly. Being defined this analysis as a tool that seeks to describe the existing patterns in spatial data and establish, preferably quantitatively, the relationships between the different geographic variables6.

One of the tools applied to perform the analyses was the Kernel density estimator, and in this study the Gaussian function with a radius of foster neighborhood, automatically defined by the software used, was used. The intensity Kernel density estimator is an exploratory technique that allows analyzing the behavior of points and estimating the punctual intensity of processes throughout the studied area7.

This non-parametric technique, in addition to estimating the intensity of the occurrence of cases throughout the analyzed surface, allows filtering the variability of a data set, while retaining its first localcharacteristics7.

The Department of Health Surveillance in all epidemiological analyses adopts an incidence radius of $900 \mathrm{~m}$, so in the generation of maps the same value was adopted for the radius of influence.

The algorithmic function applied was quadratic with grid options over the region with 200 columns presenting the adaptive type density calculation (Grid dimension - width 0.165966, height 0.151151, Resolution X 8.2983 E - 005, Resolution Y 7.55755 E - 005); point-shaped event; total events 1,800 .

The fields of patio and number were used for georeferencing. The data about neighborhood that is in the SINAN database refers to the neighborhood declared by the patient at the time of filling out the individual notification form (FIN) and, due to borderline patios, often does not represent the correct neighborhood of residence, not configuring a valid variable for georeferencing.

For non-localized cases, manual georeferencing was performed, which consisted of searching for each address on
Google Maps, Google Earth or the Post Office website.

To verify whether the loss in dengue cases influenced the spatial distribution obtained in relation to non-dengue cases, a simulation of loss in dengue cases equal to that occurred in non-dengue cases was performed. This simulation was carried out excluding from the database the difference between the percentage of severe cases existing in each neighborhood in relation to georeferenced cases.

\section{Results and Discussion}

During 2015, 2,203 dengue cases were reported throughout the municipality of Vitória de Santo Antão, of which 166 reported cases did not present completion of the field for clinical data /signs and symptoms; 114 reported cases did not present completion or ignored or white scans of the race/color field; 07 cases notified as inconclusive, 01 notified case did not present the completion of the progress date field; 267 reported cases did not present completion or ignored of the pregnancy field.

To make the identification of the reported cases, cleaning was performed in the database, excluding duplicates with the help of the SPSS program, merge function. Of the total of 2,203 autocontine dengue cases occurring in the urban and rural areas of the municipality between January and December 2015, 1,648 reported cases (1,005 reported and confirmed cases as dengue and 643 reported cases not confirmed for dengue) were selected for this study. The reasons for the non-geocoding of 403 cases (18.294\%) were no address in the notification form or inadequacy of the address with the cartographic basis.

Although a spatial distribution of cases is observed throughout the territorial extension of the municipality, figure 1 (A) shows the hot areas (areas with high intensity) in the respective neighborhoods for dengue with laboratory confirmation with $\mathrm{n}=128$ : Bela Vista and Matriz. The neighborhoods Cajá, Maués, Livramento have areas considered medium (areas with medium intensity) for dengue with laboratory confirmation. The uncited neighborhoods have low areas (areas with low intensity) for dengue with laboratory confirmation. It is observed that the hot areas are in or near subnormal clusters.

Figure 1 (B) shows the hot areas (areas with high intensity) in the respective neighborhoods for dengue with confirmation for the clinical criterion - epidemiological with $\mathrm{n}=1,027$ : Cajá, Mangueira, Lídia, Nossa Senhora do Amparo, Bela Vista. The neighborhoods Matriz, Redenção, Santana, Conceição, Maranhão, Livramento, Alto José Leal have areas considered medium (areas with medium intensity) for dengue with confirmation for the clinical criterion epidemiological. The uncited neighborhoods have low areas (areas with low intensity) for dengue with laboratory confirmation.

Figure 2 (A) demonstrates hot areas (areas with high intensity) in the respective neighborhoods for dengue with economically active population with $n=857$ : Bela Vista, Cajá, Redenção, Livramento and Mangueira. The neighborhoods 
4 Lima, A.F.P.S.; Silva, E.T.B.; Silva, E.B.; Lima, J.B.A.S.; Silva, R.M.M.; Silva, G.E.R.; Neto, J.S.V.; Alves, S.M.F.; Bezerra, J.G.; Silva, V.B.; Leite, A.G.B.; Bacelar, E.S.; Damazio, S.L.C.; Martins, I.C.; Leite, A.F.B.; Álvares, E.B.S.O. Analysis of the Spatial-Temporal Distribution of Dengue Case Notifications in the Municipality of Vitória de Santo Antão-PE...

Santana, Conceição, Maués, Matriz and Lídia Queiroz have areas considered medium (areas with medium intensity) for dengue with economically active population. The uncited neighborhoods have low areas (low intensity areas) for dengue with an economically active population.

Figure 2 (B) presents hot areas (areas with high intensity) in the respective neighborhoods for dengue with economically non-active population with $n=515$ : Lídia Queiroz, Nossa Senhora do Amparo, Bela Vista, Cajá, Mangueira. Santana and Conceição neighborhoods have areas considered medium (areas with medium intensity) for dengue with economically non-active population. The uncited neighborhoods have low areas (low intensity areas) for dengue in the economically active female population.

Figure 3 (A) found that there are hot areas (areas with high intensity) in the respective neighborhoods for dengue in the economically active male population with $n=432$ : Redemption, Deliverance and Our Lady of Amparo. The neighborhoods Água Branca, Cajá, Conceição, Matriz, Bela Vista, Maranhão, Lídia Queiroz and Maués have areas considered medium (areas with medium intensity) for dengue in the economically active male population. The uncited neighborhoods have low areas (areas with low intensity) for dengue in the economically active male population.

Figure 3 (B) in turn illustrates hot areas (areas with high intensity) in the respective neighborhoods for dengue in the economically non-active male population with $n=242$ : Lydia Queiroz, Livramento, Nossa Senhora do Amparo, Mangueira and Bela Vista. The neighborhoods Água Branca, Conceição, Cajá, Matriz, Maranhão, Jardim Ipiranga have areas considered average (areas with medium intensity) for dengue in the non-active male population. The uncited neighborhoods have low areas (areas with low intensity) for dengue in the economically active male population.

Figure 4 (A) presents hot areas (areas with high intensity) in the respective neighborhoods for dengue in the economically active female population with $n=425$ : Cajá, Mangueira, Lídia Queiroz, Nossa Senhora do Amparo, Bela Vista, Santana, Conceição and Redenção. Maués, Livramento neighborhoods have areas considered medium (areas with medium intensity) for dengue in the economically active female population. The uncited neighborhoods have low areas (low intensity areas) for dengue in the economically active female population.

Figure 4 (B) shows hot areas (areas with high intensity) in the respective neighborhoods for dengue in the economically non-active female population with $n=273$ : Cajá, Conceição, Lídia Queiroz, Nossa Senhora do Amparo, Livramento, Jardim Ipiranga, Bela Vista, Alto José Leal. The neighborhood of Água Branca has areas considered medium (areas with medium intensity) for dengue in the economically non-active female population. The uncited neighborhoods have low areas (low intensity areas) for dengue in the economically active female population.

Figure 5 (A) in turn shows the presence of hot areas (areas with high intensity) in the respective neighborhoods for dengue in the black population with $n=1,178$ : Água Branca, Conceição, Cajá, Lídia Queiroz, Nossa Senhora do Amparo, Livramento. The neighborhoods Redenção, Matriz, Maués, Bela Vista and Jardim Ipiranga have areas considered medium (areas with medium intensity) for dengue in the black population. The uncited neighborhoods have low areas (areas with low intensity) for dengue in the black population.

Figure 5 (B) indicates the existence of hot areas (areas with high intensity) in the respective neighborhoods for dengue in the non-black population with $n=194$ : Redemption, Bela Vista and Cajá. The neighborhoods Lídia Queiroz, Mangueira and Livramento have areas considered medium (areas with medium intensity) for dengue in the black population. The uncited neighborhoods have low areas (areas with low intensity) for dengue in the black population.

Figure 6 (A) demonstrates hot areas (areas with high intensity) in the respective neighborhood for dengue with Classification level 1 - Less and equal to 5 signs and symptoms, except signs and symptoms defined by the Ministry of Health as severe with $n=1,112$ : Nossa Senhora do Amparo. The neighborhoods Conceição, Cajá, Redenção, Matriz, Bela Vista, Jardim Ipiranga and Lídia Queiroz have areas considered average (areas with medium intensity) for dengue Classification level 1. The uncited neighborhoods have low areas (areas with low intensity) for dengue with level 1 classification. Thus, we can affirm that the neighborhood that displays the punctual intensity of the process that presents dengue with Classification level 1 is the neighborhood of Nossa Senhora do Amparo.

Figure 6 (B) showed the presence of hot areas (areas with high intensity) in the respective neighborhoods for dengue with classification level 2 - Greater than 5 symptoms or with severity bells and deaths with $n=257$ : Conceição, Água Branca, Cajá, Redenção, Lídia Queiroz, Nossa Senhora do Amparo, Livramento, Mangueira and Bela Vista. The neighborhoods Maranhão, Matriz and Maués have areas considered medium (areas with medium intensity) for dengue Level 2 classification. The uncited neighborhoods have low areas (areas with low intensity) for dengue with level 2 classification. Thus, we can affirm that the neighborhood that displays the punctual intensity of the process that presents dengue with Classification level 2 are the neighborhoods Conceição, Água Branca, Cajá, Redenção, Lídia Queiroz, Nossa Senhora do Amparo, Livramento, Mangueira and Bela Vista.

It is concluded that dengue cases are not random, but can be considered grouped, that is, the phenomenon does not occur randomly, being possible to affirm that there is a spatial trend. The applications of spatial analysis techniques obtained satisfactory results regarding the behavior of dengue incidence throughout 2015.

Regarding kernel maps, they identified the areas with strong concentrations throughout 2015 and it can also be concluded that dengue in the municipality of Vitória de Santo Antão is related to socioeconomic and infrastructure issues, because the neighborhoods Cajá, Mangueira, Lídia Queiroz, Nossa Senhora do Amparo present hot areas for dengue 
(FIGURE 5 (A)) with a Lower Social Development Index (IDS) than that recorded in the municipality of Vitoria of Santo Antão.

In the evaluation of the losses generated by the georeferencing process, there was no loss in dengue records $(100 \%)$, the spatial distribution remained unchanged, not harming the results previously presented.

Through these analyses it can be concluded that dengue in the municipality of Vitória de Santo Antão is related to socioeconomic and infrastructure issues, because the neighborhoods Cajá, Mangueira, Lídia Queiroz, Nossa Senhora do Amparo present hot areas for dengue (FIGURE 5 (A)) with a Lower Social Development Index (IDS) than that registered in the municipality of Vitória de Santo Antão.

Of the 28 neighborhoods in the municipality of Vitória de Santo Antão, 16 presented kernel density between medium and high for dengue cases. This shows that dengue is distributed geographically throughout the municipality. The Kernel ratio was calculated for the reported cases of dengue from the estimation of the density of the cases and the population, and an approximation of the risk areas was performed, since their values are weighted by the casecontrol relationship.

Spatial analysis has been used for many years in exact sciences to verify the dispersion of soil contamination, extension of mineral deposits and the variation of geoenvironmental characteristics of regionalized data3. The results presented in this research demonstrate that spatial georeferencing can also be used as a tool in the identification of areas at risk of dengue transmission, and can be applied and directed by public managers.

The isolated representation presented in all figures provides a graphic image of the situation of the epidemic, being visualized in the form of clouds or continuous spots that spread throughout the geographic space as particular morphology and orientation for each variable applied in the study: dengue with laboratory confirmation; dengue with confirmation for the clinical - epidemiological criterion; with economically active population; economically non-active population; dengue in the economically active male population; dengue in the economically non-active male population; dengue in the economically active female population; dengue in the economically non-active female population; dengue in the black population; dengue in the non-black population; dengue with Classification level 1 (Less and equal to 5 signs and symptoms, except signs and symptoms defined by the Ministry of Health as severe); dengue with Level 2 classification (Level 2 classification Greater than 5 symptoms or with severity bells and deaths).

These findings show that in the neighborhoods Cajá, Mangueira, Lídia Queiroz, Nossa Senhora do Amparo, regardless of the central region concentrate most of the resident population, dengue is spread throughout the city. This fact is possibly justified in the municipality, due to the location of the residences on the periphery and the distance from the workplace, which allows an intense flow from one place to another, also to the lack of awareness of the community itself, which, even instructed, does not take the proper precautions with the breeding sites of vector proliferation, in addition to the difficulties encountered by sanitary and epidemiological surveillance in the control and elimination of mosquito larvae in wastelands. Corroborating our study, Tannous8 states that the control of the proliferation of Aedes aegypti has become a difficult task due to disorderly urbanization, the difficulty of cleaning urban centers, the ease of movement and locomotion of the population within cities, in addition to the concomitant ineffectiveness of policies to combat the mosquito associated with lack of population awareness.

Figure 2 (A - B), figure 3 (A - B) and figure 4 (A - B), which were occurred, which were observed in these studies, which were the case of disorderly urbanization. According to reports, there is a trend that occurs throughout the territorial area related to the endemic-epidemic process of dengue. It is evidenced in large regions, mainly due to the process of disordered urbanization that results in regions with high population density, areas with precarious public services related to urban infrastructure and other factors9.

Figure 6 (B) showed the presence of hot areas (areas with high intensity) in the respective neighborhoods for dengue with classification level: Conceição, Água Branca, Cajá, Redenção, Lídia Queiroz, Nossa Senhora do Amparo, Livramento, Mangueira and Bela Vista. In reference to the study by Carvalho, Magalhães and Medronho7, the results found suggest that barriers to access to health services interfere in the early diagnosis of dengue, leading to the greater possible possible cases ofsevere dengue, since the late diagnosis of the disease may imply the non-identification of the alarm signs of the disease. It is noteworthy that the improvement of access to health services is related to the principle of universality provided for in the Unified Health System.

It is noteworthy that knowledge of the geographic distribution of dengue is essential for understanding its contribution to global morbidity burdens of mortality, in determining available resources for dengue control and in assessing the impact of these activities. In addition, estimates and distributions of unapparent infection form a fundamental requirement to assess clinical surveillance, demands and future strategies 10 .

We can conclude that areas with high intensity are not taken into account when planning control actions, because they are less developed areas. On the other, areas with areas with medium intensity are "better neighborhoods". For this reason, a new look should be launched on these areas with high intensity, being able to assist in decision making, taking into account the stratification by degrees of risk, which will certainly contribute to the planning of measures and applications of control actions reducing costs and response time in the event of epidemics.

\section{Conclusion}


6 Lima, A.F.P.S.; Silva, E.T.B.; Silva, E.B.; Lima, J.B.A.S.; Silva, R.M.M.; Silva, G.E.R.; Neto, J.S.V.; Alves, S.M.F.; Bezerra, J.G.; Silva, V.B.; Leite, A.G.B.; Bacelar, E.S.; Damazio, S.L.C.; Martins, I.C.; Leite, A.F.B.; Alvares, E.B.S.O. Analysis of the Spatial-Temporal Distribution of Dengue Case Notifications in the Municipality of Vitória de Santo Antão-PE...

The analysis of the spatial-temporal distribution of case notifications, if performed constantly throughout the year, can direct public policies to more direct actions to control and combat the disease vector. The space-time approach, through specialized software, allows the integration of demographic, economic and environmental data, promoting the interrelationship of information and various databases.

In this context, spatial analysis is shown as a tool that can be applied in several spheres of health, as in spatial and temporal diffusion studies. Thus, it allows to observe areas with higher epidemiological risk, both by locating foci with potential for vector proliferation, Aedes aegypti, as well as social and demographic factors, which inevitably predict the occurrence of the disease.

\section{Ethical aspects}

This research was approved by the Research Ethics Committee involving human beings of the Center for Health Sciences of UFPE.

Data were collected after approval of this body. The technique applied in this research was to search for secondary data in SINAN.

The research sought to meet the recommendations of Resolution No. 466/12 of the National Health Council/Ministry of Health - CNS/MS, which provides for the standards applicable to research in Human and Social Sciences whose methodological procedures involve the use of data directly obtained from participants or identifiable information or that may entail greater risks than those existing in everyday life, in the defined form found in the above-mentioned Resolution.

\section{Acknowledgments}

To the Department of Health Surveillance of the Municipality of Vitória de Santo Antão, PE.

\section{References}

[1] Skalinski LM, Costa MCN, Teixeira MGL. Contribuições da análise espacial para a compreensão da dinâmica de transmissão da dengue: revisão integrativa. J. Health Biol. Sci. 2019;7(1).

[2] Camargo DM, Souza NM, Pacheco TCKF, Alcântara GC, Dota EM. Modelagem geoespacial para identificação de áreas vulneráveis ao contágio por doenças relacionadas a falta de saneamento: o caso da região metropolitana de Campinas. Revista Brasileira de Cartografia, 2017;69/3:561-573.

[3] Cavalcante MPR. et al. Análise geoespacial: um estudo sobre a dengue. Acta Paul Enferm. 2013;26(4).

[4] Brasil. Ministério da Saúde. Secretaria de Vigilância em Saúde. Monitoramento dos casos de dengue, febre de chikungunya e febre pelo vírus Zika até a Semana Epidemiológica 3. Bol Epidemiol. 2016a;47(6):1-7.

[5] Brasil. Ministério da Saúde. Secretaria de Vigilância em Saúde. Monitoramento dos casos de dengue, febre de Chikungunya e febre pelo vírus Zika até a Semana Epidemiológica 37. Bol Epidemiol. 2016b;47(34):1-10.

[6] Carvalho S, Magalhães MAFM, Medronho RA. Análise da distribuição espacial de casos da dengue no município do Rio de Janeiro, 2011 e 2012. Rev. Saúde Publica. 2017; 51:1-10.

[7] Tannous IP, perfil epidemiológico e geográfico da infecção pelo vírus da dengue em um município do sudoeste de Goiás: um estudo transversal. 2018.

[8] Mendes JS, Meliani PF, Spanghero PESF. Dengue na Bahia: análise da dinâmica espaço-temporal no período de 2001 a 2010. Cam. Geo. 2017;18(62):73-86.

[9] Pellissari BP. et al. Aspectos Socioambientais associados à ocorrência de Dengue em um Município do estado do Mato Grosso. Rev. Epidemiol. Controle Infecç. 2016;6(1).

\section{Attachments}

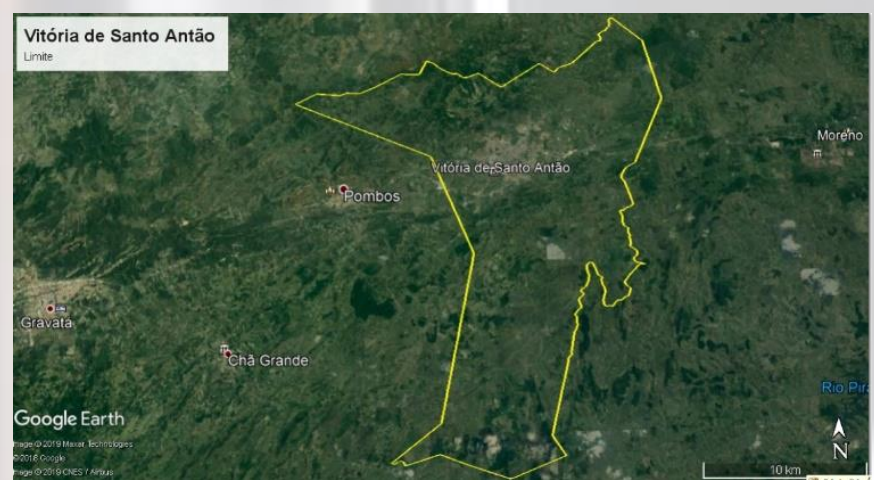

Figure 1. Geographical location of the municipality of Vitória de Santo Antão - PE. Source: Google Earth Pro Platform

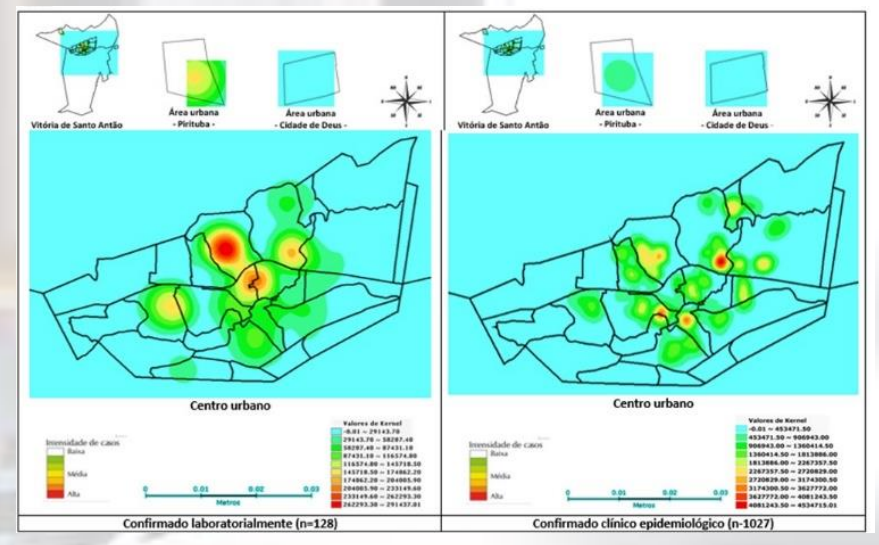

Figure 2. Map and kernel parameters of dengue cases according to case confirmation criterion classification, Vitória de Santo Antão, 2015. Source: Prepared by the author based on data from the Notifiable Diseases Information System (SINAN) 


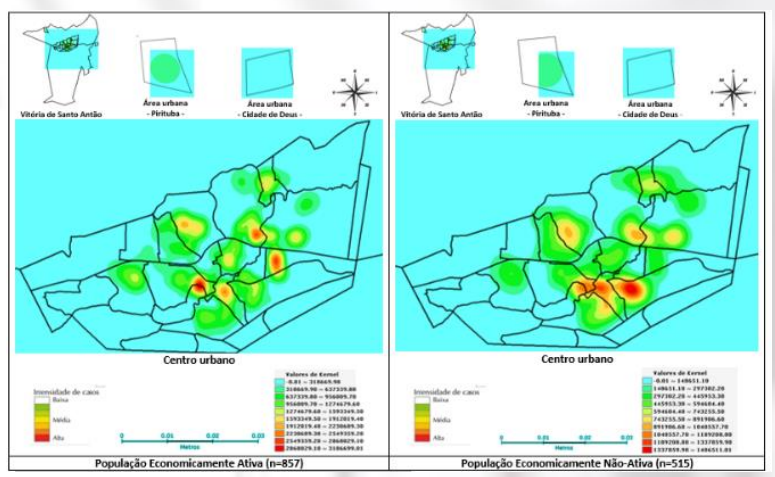

Figure 3. Map and kernel density parameter of dengue reported case according to economically active population. Vitória de Santo Antão, 2015. Source: Prepared by the author based on data from the Notifiable Diseases Information System (SINAN). Note: Economically active population $=$ age 10 to 65 years

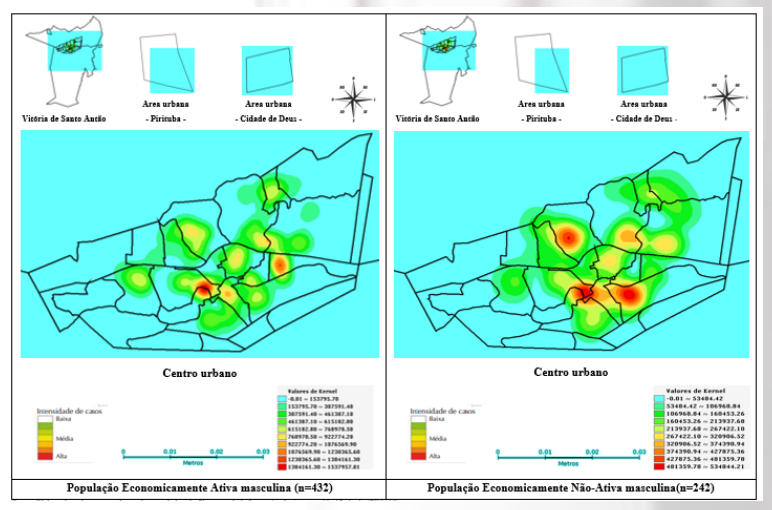

Figure 4. Map and kernel density parameter of dengue case reported according to economically active male population. Vitória de Santo Antão, 2015. Source: Prepared by the author based on data from the Notifiable Diseases Information System (SINAN). Note: Economically active population = age 10 to 65 years

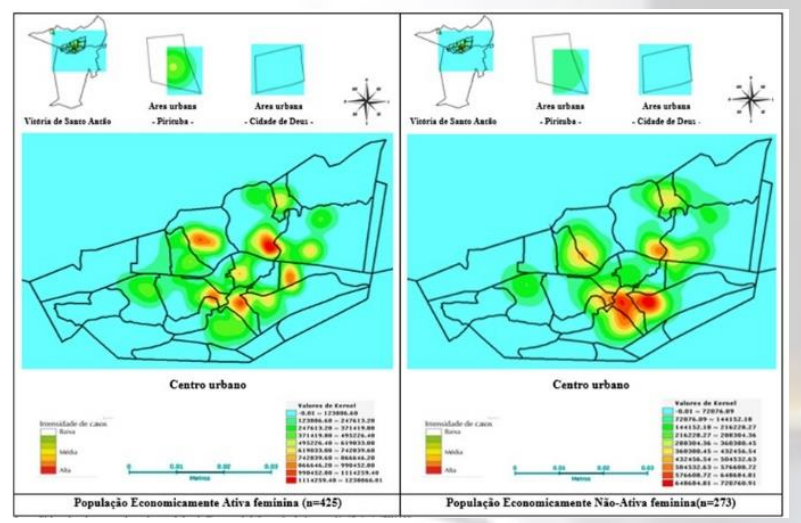

Figure 5. Map and kernel density parameter of dengue reported case according to economically active female population. Vitória de Santo Antão, 2015. Source: Prepared by the author based on data from the Notifiable Diseases Information System (SINAN). Note: Economically active population $=$ age 10 to 65 years

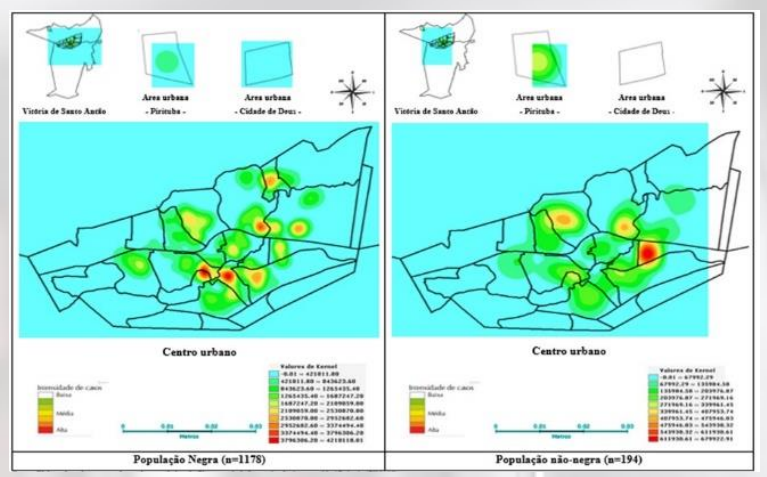

Figure 6. Map and kernel density parameter of dengue case notified according to breed. Vitória de Santo Antão, 2015. Source: Prepared by the authors based on data from the Notifiable Diseases Information System (SINAN)

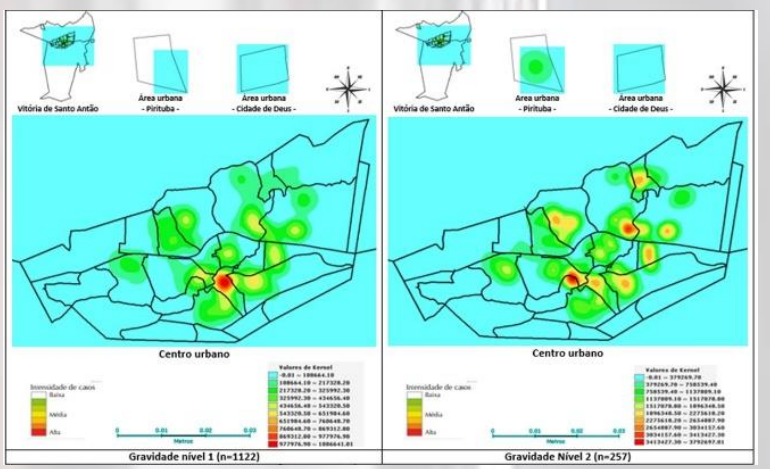

Figure 7. Map and kernel density parameter of dengue case reported according to severity of cases, Vitória de Santo Antão, 2015. Source: Prepared by the author based on data from the Notifiable Diseases Information System (SINAN). Note: Sum of presence and absence of clinical signs and symptoms.Level 1 Rating - Less than and equal to 5 signs and symptoms, except signs and symptoms defined by the Ministry of Health as severe. Level 2 classification - Greater than 5 symptoms or with severity bells and deaths. 